\title{
Atara Biotherapeutics
}

National Cancer Institute

\section{Source}

National Cancer Institute. Atara Biotherapeutics. NCI Thesaurus. Code C134822.

A privately-held drug development company that is developing cancer treatments based on allogeneic cytotoxic T lymphocyte immunotherapy. 